\title{
Drug resistance in cancer therapy
}

Vinod Labhasetwar

Published online: 12 November 2011

(C) Controlled Release Society 2011

The risk of tumors acquiring resistance to cancer chemotherapeutics remains the major obstacle to successful treatment of many cancers [1]. Ironically, such resistance is not only to the drug the cancer cells have been exposed to, but also to many other structurally unrelated anticancer drugs; this multidrug resistance makes treating such tumors more difficult [2]. To avoid higher doses of anticancer drugs with unacceptable toxicity, patients may receive a "subtherapeutic" dose that causes tumors acquire resistance. In general, cancer cells develop resistance due to (a) changes in cell membrane-associated factors that control influx/efflux of drugs, (b) accumulation of drugs in intracellular vesicles or change in their physical form once inside cells so they either become unavailable or cannot bind to their intracellular targets, (c) drug instability inside cancer cells because of intracellular environmental changes, and/or (d) changes in cells' nuclear and genetic factors. In addition to acquired drug resistance, certain cancer cells exhibit intrinsic resistance to commonly used drugs because of genetic/epigenetic changes [3].

The general approach is to invade the cellular and molecular mechanisms by which tumor cells develop drug resistance [4-7]. Different strategies to overcome resistance include: (a) using a drug "cocktail" that acts on more than one molecular pathway; (b) developing polymer conjugates and novel-targeted nanocarrier drug delivery systems to overcome cell/tissue barriers; and more recently, (c) using small interfering RNAs and oligonucleotides to specifically silence genes or gene products responsible for drug resistance. The most common drug delivery approach is to use nanocarriers such as nanoparticles, liposomes, or dendrimers, with or without a targeting ligand (e.g., antibody, peptides, or aptamers) against the receptors or antigens

V. Labhasetwar $(\square)$

Department of Biomedical Engineering/ND20,

Lerner Research Institute, Cleveland Clinic,

Cleveland, OH 44195, USA

e-mail: labhasv@ccf.org overexpressed in cancer cells (e.g., transferrin, folic acid, luteinizing hormone-releasing hormone, or epidermal growth factor) to improve drug localization to tumor tissue, through passive/active targeting mechanisms [6].

In our recent study, we showed significant differences in the lipid composition of doxorubicin-sensitive (MCF-7) and doxorubicin-resistant (MCF-7/ADR) breast cancer cells that do not only influence their biophysical properties, particularly membrane lipid packing and fluidity, but also drug interaction with lipids and transport. The lipids of resistant cells form more condensed and less fluid monolayers than the lipids of sensitive cells. In addition, the lipid-doxorubicin interactions in resistant cells decrease intracellular drug transport via diffusion as the drug is trapped in the lipid bilayer. The rigid nature of resistant cell membranes also seems to influence endosomal functions that inhibit drug uptake when a liposomal formulation of doxorubicin was used [8]. Hence, nanocarriers, which can evade the cell membrane barrier and/or escape rapidly and efficiently from the endosomal compartment, could be effective in enhancing the intracellular drug delivery and overcoming drug resistance. However, as described above, there are multiple mechanisms by which cancer may acquire drug resistance and it would also depend upon type of tumors. Therefore, a methodological analysis of the biology of drug resistance and according developing drug delivery strategy could be effective.

In this issue of the journal, Professor Mahato's group has shown that lapatinib, which interacts with and inhibits pgycoprotein (P-gp) activity, when used in combination with paclitaxel can synergistically treat resistant prostate cancer. P-gp is a membrane-associated efflux transporter, which influences the intracellular retention of anticancer drugs in resistant cells. In a xenograft athymic nude mice tumor model, the group has shown significant tumor growth inhibition when the combination of lapatinib and paclitaxel was given twice a week intravenously using a micellar formulation. The combination therapy synergistically decreased antiangiogenic activity compared to the control or their monotherapy. 
Cancer nanomedicine is an important field where nanotechnology is used to deliver drugs more effectively than conventional formulations which results in significant toxicity. The recent approaches include developing theranostic agents with a combined drug delivery and imaging properties $[9,10]$. Such systems could allow monitoring drug distribution in real time to ensure that therapeutic doses of drugs are delivered to tumor tissue. In recent years, advances in early detection techniques and improved treatment options have led to cures in the early stages of several cancers; however, effective therapies for late stage and more aggressive forms of the disease are still greatly needed. In addition, the major challenge is treating metastatic cancers. Nanomedicine has potential to develop effective cancer treatments, but basic understanding of the tumor biology is also needed for such efforts.

\section{References}

1. Kuo MT. Redox regulation of multidrug resistance in cancer chemotherapy: Molecular mechanisms and therapeutic opportunities. Antioxid Redox Signal. 2009;11(1):99-133.
2. Morrow CS, Cowan KH. Mechanisms and clinical significance of multidrug resistance. Oncology (Williston Park). 1988;2(10):5563.

3. Savage P, Stebbing J, Bower M, Crook T. Why does cytotoxic chemotherapy cure only some cancers? Nat Clin Pract Oncol. 2009;6(1):43-52.

4. Blanco E, Kessinger CW, Sumer BD, Gao J. Multifunctional micellar nanomedicine for cancer therapy. Exp Biol Med (Maywood). 2009;234(2):123-31.

5. Singh Y, Palombo M, Sinko PJ. Recent trends in targeted anticancer prodrug and conjugate design. Curr Med Chem. 2008;15(18):1802-26.

6. Begley J, Ribas A. Targeted therapies to improve tumor immunotherapy. Clin Cancer Res. 2008;14(14):4385-91.

7. Cho K, Wang $X$, Nie S, Chen ZG, Shin DM. Therapeutic nanoparticles for drug delivery in cancer. Clin Cancer Res. 2008;14(5):1310-6.

8. Peetla C, Bhave R, Vijayaraghavalu S, Stine A, Kooijman E, Labhasetwar V. Drug resistance in breast cancer cells: Biophysical characterization of and doxorubicin interactions with membrane lipids. Mol Pharm. 2010;7(6):2334-48.

9. Foy SP, Manthe RL, Foy ST, Dimitrijevic S, Krishnamurthy N, Labhasetwar V. Optical imaging and magnetic field targeting of magnetic nanoparticles in tumors. ACS Nano. 2010;4(9):5217-24.

10. Jain TK, Foy SP, Erokwu B, Dimitrijevic S, Flask CA, Labhasetwar V. Magnetic resonance imaging of multifunctional pluronic stabilized iron-oxide nanoparticles in tumor-bearing mice. Biomaterials. 2009;30(35):6748-56. 